\title{
A Mathematical Study of the One-Dimensional Keller and Rubinow Model for Liesegang Bands
}

\author{
D. Hilhorst • R. van der Hout $\cdot$ M. Mimura $\cdot$ I. Ohnishi
}

Received: 2 September 2008 / Accepted: 23 February 2009 / Published online: 14 March 2009

(C) The Author(s) 2009. This article is published with open access at Springerlink.com

\begin{abstract}
Our purpose is to start understanding from a mathematical viewpoint experiments in which regularized structures with spatially distinct bands or rings of precipitated material are exhibited, with clearly visible scaling properties. Such patterns are known as Liesegang bands or rings. In this paper, we study a one-dimensional version of the Keller and Rubinow model and present conditions ensuring the existence of Liesegang bands.
\end{abstract}

Keywords Reaction-diffusion $\cdot$ Liesegang bands $\cdot$ Scaling laws $\cdot$ Nonlocal terms

\section{Introduction}

In 1896, colloid-chemist R.E. Liesegang [6] observed strikingly regular patterns in precipitation-reaction processes, which are referred to as Liesegang bands or rings, according to their shape. These precipitation reactions are briefly described as follows. A solution of soluble electrolyte $\left(\mathrm{Pb}\left(\mathrm{NO}_{3}\right)_{2}\right.$, say) at relatively low concentration is placed in a test tube which is occupied by gel. Then a second electrolyte (say KI) at high concentration is added on top of the gel so that $\mathrm{I}^{-}$ions diffuse into the gel and react with $\mathrm{Pb}^{+}$ions to form $\mathrm{PbI}_{2}$, which is insoluble. Then layers of $\mathrm{PbI}_{2}$-precipitate appear, with fronts parallel to the surface

D. Hilhorst

Laboratoire de Mathématique, CNRS and Université de Paris-Sud XI, Bât. 425, 91405 Orsay Cedex, France

R. van der Hout $(\bowtie)$

Faculty of Science, VU University, De Boelelaan 1081 A, 1081 HV Amsterdam, The Netherlands e-mail: rein.vanderhout@planet.nl

M. Mimura

Department of Mathematics, School of Science and Technology Meiji Institute for Mathematical

Science and Technology, Meiji University, Higashimita, Tama-ku, Kawasaki, 214-8571 Japan

I. Ohnishi

Dept. of Mathematical and Life Sciences, Graduate School of Science, Hiroshima University,

Higashi-Hiroshima, 739-8526 Japan 


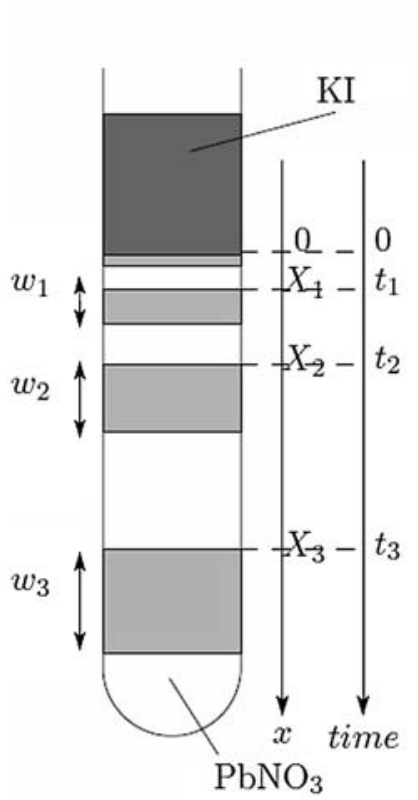

$$
\begin{aligned}
\frac{X_{n+1}}{X_{n}} & =\text { const. } & & {[\text { spacing law }] } \\
\frac{X_{n}}{\sqrt{t_{n}}} & =\text { const. } & & {[\text { time law }] } \\
\frac{w_{n}}{X_{n}} & =\text { const. } & & {[\text { width law }] }
\end{aligned}
$$

Fig. 1 Precipitation fronts and scaling laws

of the diffusion front, as it is shown in the left-hand part of Fig. 1; this figure also shows a pattern of Liesegang rings in a radially symmetric situation.

These precipitate-layers exhibit several scaling properties. If we denote by $X_{n}$ the distance from the $n$-th band location to the first one, then it is surprising to observe that $X_{n+1}=\sigma X_{n}$ for some positive constant $\sigma$. This phenomenon is the so called spacing law. In addition, it has been observed that there exists a positive constant $\alpha$ such that $X_{n}=\alpha \sqrt{t_{n}}$, where $t_{n}$ is the time at which the $n$-th band germinates. This relation is generally referred to as the time law. Finally, if $w_{n}$ denotes the thickness of the $n$-th layer, it appears that $w_{n+1} / w_{n}$ is approximately constant: this is the so-called width law. We refer to Fig. 2 below for a sketch of a possible configuration; however this figure neither represents a real experiment nor the result of a numerical simulation.

In order to understand the mechanism behind these laws, intensive studies have been done, both from experimental and from theoretical viewpoints (see [7] and the references therein). Two important theories have been developed. The first one is the pre-nucleation theory, which is based upon super-saturation ideas [9]: in this context a reaction-diffusion model was proposed by Keller and Rubinow [5]. This is the model which we will study in this paper. Numerical experiments for this model clearly show one-dimensional bands which satisfy the spacing law; however ring patterns do not seem to occur in radially sym- 
Fig. 2 Illustration of the time-law

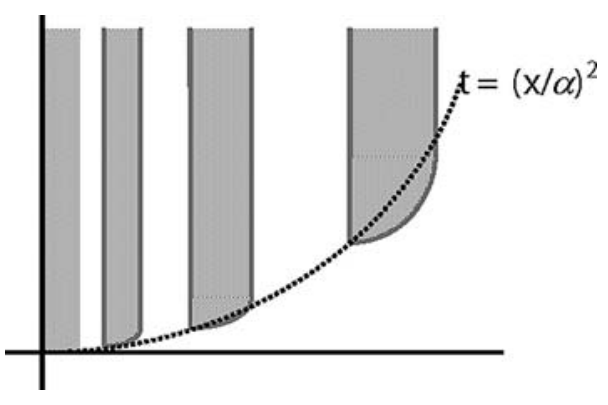

metric situations. In other words, the Keller and Rubinow model is not valid in higher space dimension. The second theory is the post-nucleation theory, which is based upon the Ostwald's ripening process for colloidal particles. This theory includes instability and competitive growth theory. In two space dimensions, the proposed model, which describes the dynamics of colloidal particles and includes nucleation effects, does not only exhibit rings but also spiral patterns [4].

The object of this paper is to theoretically discuss the existence of discrete precipitation bands arising in experiments. In order to do so, we choose a one-dimensional model, based upon the ideas of Keller and Rubinow. However results of numerical simulations clearly show that this model does not permit to exhibit the width law. Let $a, b$ and $c$ be the concentrations of the monomers $\mathcal{A}, \mathcal{B}$ and the product $\mathcal{C}$ for the reaction process $\mathcal{A}+\mathcal{B} \rightarrow \mathcal{C}$ and let $d$ be the concentration of the precipitate $\mathcal{D}$, formed by the precipitation $\mathcal{C} \rightarrow \mathcal{D}$. Then the processes in one space dimension can be described by the following reaction-diffusion system:

$$
\left\{\begin{array}{l}
a_{t}=D_{a} a_{x x}-k a b, \\
b_{t}=D_{b} b_{x x}-k a b, \\
c_{t}=D_{c} c_{x x}+k a b-P(c, d), \\
d_{t}=P(c, d)
\end{array}\right.
$$

where $D_{a}, D_{b}$ and $D_{c}$ are respectively the diffusivities of $A, B$ and $C . P(c, d)$ is the precipitation term which includes a super-saturation effect. It is given by the following expression:

$$
P(c, d)= \begin{cases}0 & \text { if } c<C_{s} \text { and } d=0, \\ \lambda\left(c-c^{*}\right)^{+} & \text {if } c \geq C_{s} \text { or } d>0,\end{cases}
$$

where $C_{s}$ and $c^{*}\left(C_{s}>c^{*}\right)$ are the super-saturation and saturation concentrations, respectively; $\lambda$ is the rate constant of the precipitation $\mathcal{C} \rightarrow \mathcal{D}$. The superscript ${ }^{+}$denotes nonnegative parts. In this paper, we simplify (1), under the following assumptions:

(i) The diffusion rate $D_{b}$ is much smaller than $D_{a}$.

(ii) The reaction rate $k$ is very large.

(iii) $c^{*}=0$.

(iv) The processes occur in a semi-infinite slab $0<x<\infty$.

Assumption (i) permits to perform a number of explicit computations. Without this assumption, we can formally perform similar calculations, but we have no proof of their validity. Assumption (ii) is realistic from a chemical view point. 
Fig. 3 Concentration profiles, appropriately scaled
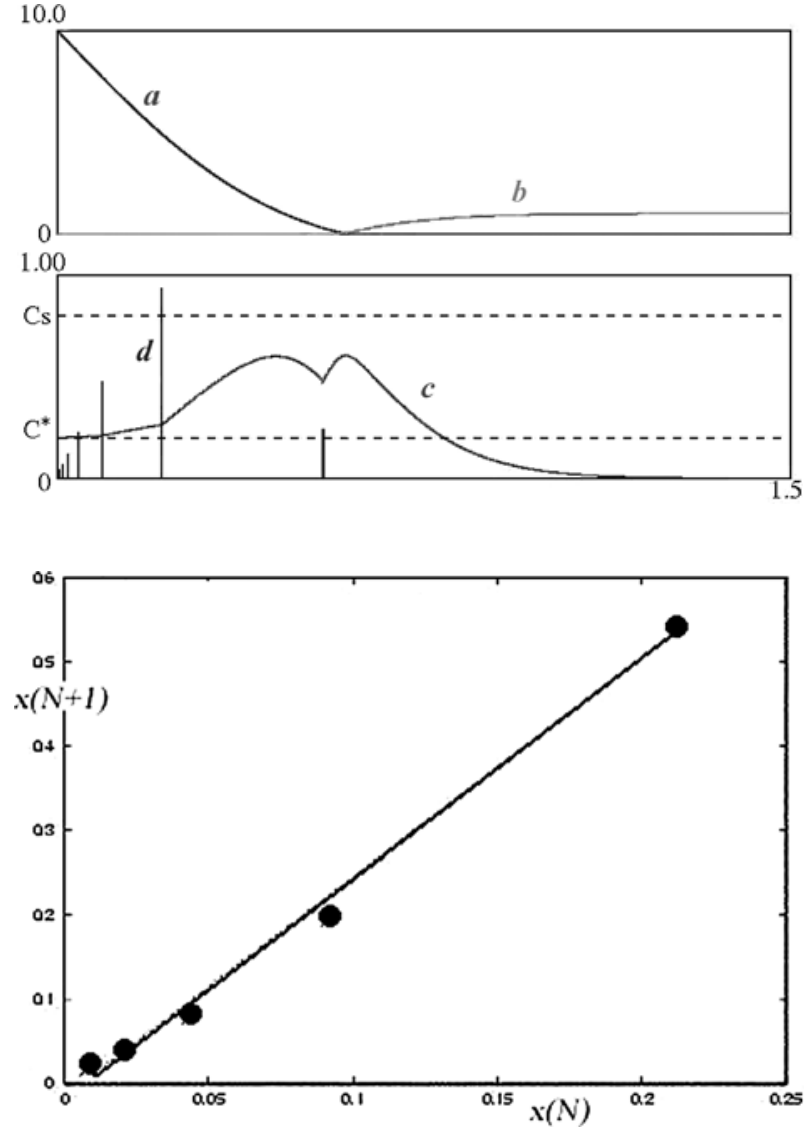

Fig. 4 Simulation results: spacing law, appropriately scaled

This leads to the simplified system

$$
\begin{cases}a_{t}=D_{a} a_{x x}-k a b, & 0<x<\infty, t>0, \\ b_{t}=-k a b, & 0<x<\infty, t>0, \\ c_{t}=D_{c} c_{x x}+k a b-P(c, d), & 0<x<\infty, t>0, \\ d_{t}=P(c, d), & 0<x<\infty, t>0,\end{cases}
$$

where we assume $k$ to be very large. The initial and boundary conditions read

$$
\left\{\begin{array}{l}
a(x, 0)=c(x, 0)=d(x, 0)=0, \quad b(x, 0)=b_{0}, \quad 0<x<\infty, \\
a(0, t)=a_{0}, \quad c_{x}(0, t)=0, \quad t>0,
\end{array}\right.
$$

where $a_{0}$ and $b_{0}$ are positive constants. In experiments, $a_{0}$ is much larger than $b_{0}$.

We first show some numerical simulations of (3)-(4), where, in contrast to (iii) above, we have assumed that $c^{*}>0$.

Figure 3 shows profiles of $a, b, c$ and $d$ at a certain time. Note that several precipitation events have occurred; these events are characterized by peaks of the function $d$ and corresponding discontinuities of the space derivative of the function $c$.

Figures 4 and 5 show numerical evidence of the spacing- and time laws, respectively. 
Fig. 5 Simulation results: time law, appropriately scaled

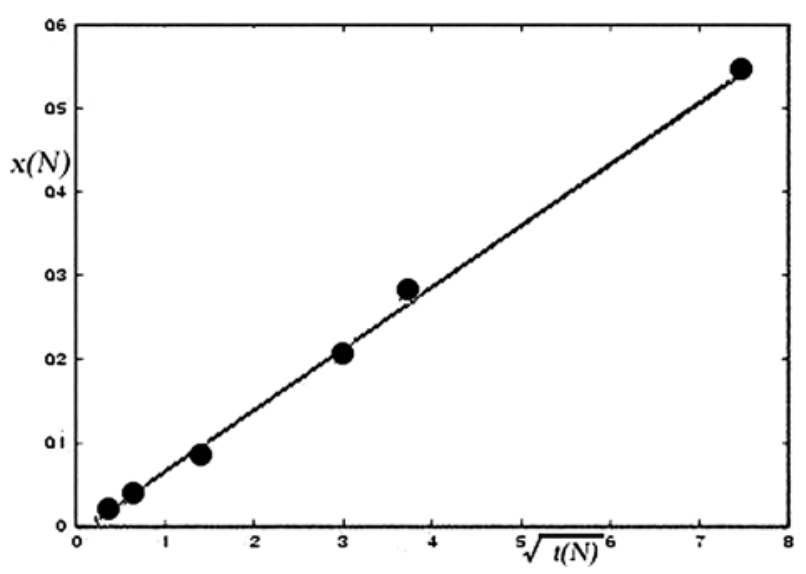

The purpose of the subsequent sections is to show that the simplified Keller-Rubinow model (3)-(4) exhibits infinitely many distinct precipitations (Theorem 3.13), under suitable conditions and assumptions, and that the time-law is satisfied. The problem formulation which we obtain turns out to involve a nonlocal discontinuous term. We prove existence of a weak solution which exhibits a bounded precipitation region (a precise definition follows later); we prove the existence of infinitely many distinct precipitation regions under an additional assumption on the behavior of the weak solution.

This paper is organized as follows. In Sect. 2, we discuss the limit as $k \rightarrow \infty$. In particular, we formulate the resulting problem for the limiting concentration $c$. Finally, in Sect. 3 , we show the conditional existence of discrete precipitation bands as well as the time law discussed above.

\section{Asymptotic Analysis}

To begin with, we rewrite Problem (3), (4), as follows, taking into account the assumption that $c^{*}=0$ :

$$
\left(\mathrm{P}_{k}^{*}\right) \begin{cases}a_{t}=D_{a} a_{x x}-k a b, & 0<x<\infty, t>0, \\ b_{t}=-k a b, & 0<x<\infty, t>0, \\ c_{t}=D c_{x x}+k a b-\lambda c \tilde{H}\left(\left(c-C_{s}\right)^{+}+d\right), & 0<x<\infty, t>0, \\ d_{t}=\lambda c \tilde{H}\left(\left(c-C_{s}\right)^{+}+d\right), & 0<x<\infty, t>0, \\ a(0, t)=a_{0}>0, & t>0, \\ c_{x}(0, t)=0, & t>0, \\ a(x, 0)=c(x, 0)=d(x, 0)=0 ; \quad b(x, 0)=b_{0}>0 & 0<x<\infty,\end{cases}
$$

where $D_{c}$ is now replaced by $D$ and where $\tilde{H}$ is the Heaviside function:

$$
\tilde{H}(y)= \begin{cases}0 & \text { when } y \leq 0 \\ 1 & \text { when } y>0\end{cases}
$$

The equations for $c$ and $d$ in Problem $\left(\mathrm{P}_{k}^{*}\right)$ have been formulated to express the chemical assumption that $d_{t}=\lambda c$ if $c>C_{s}$ or if $d>0$ and that $d_{t}=0$ otherwise. The above formulation gives rise to a non-uniqueness issue that may be avoided if we choose a different 
formulation. The right-hand sides of the equations for $c$ and $d$ are not continuous. For this reason, we cannot be sure that the solution is unique, even if $c-C_{s} \leq 0$ everywhere. Chemical arguments imply that $d(x, t)$ can only be positive if its growth has been initiated by a positive value of $c(x, \tau)-C_{s}$ for some $\tau \leq t$. This leads us to look for a solution such that $d(x, t)=0$ if $c(x, \tau) \leq C_{s}$ for all $\tau<t$. Against this background, we reformulate Problem $\left(\mathrm{P}_{k}^{*}\right)$ in a slightly different way, which is more precise from a chemical point of view, since it excludes the possibility of spontaneous growth of $d$. Assuming, for a moment, that $c$ is continuous, we introduce the function

$$
w(x, t)=\int_{0}^{t}\left(c(x, s)-C_{s}\right)^{+} d s .
$$

We claim that $w(x, t)>0$ if and only if $\left(c(x, t)-C_{s}\right)^{+}+d(x, t)>0$. Indeed let us first assume that $w(\bar{x}, \bar{t})>0$. Then there exist $t_{1}<t_{2} \leq \bar{t}$ such that $c(x, t)>C_{s}$ for all $t_{1}<$ $t<t_{2}$. The differential equation for $d$ implies that $d(\bar{x}, \bar{t})>0$, which, in turn, implies that $\left(c(\bar{x}, \bar{t})-C_{s}\right)^{+}+d(\bar{x}, \bar{t})>0$. Conversely, if $w(x, t)=0$, it is clear that $c(x, \tau) \leq C_{s}$ for all $\tau \leq t$. The chemical arguments discussed above now imply that $d(x, t)=0$.

Therefore, it is justified to replace the argument $\left(c-C_{s}\right)^{+}+d$ of $\tilde{H}$ by the new argument $w$. Note that, upon this change of arguments, any reference to $d$ can be completely omitted from Problem $\left(\mathrm{P}_{k}^{*}\right)$. We are left with the following problem, which we still refer to as Problem $\left(\mathrm{P}_{k}^{*}\right)$ :

$$
\left(\mathrm{P}_{k}^{*}\right) \begin{cases}a_{t}=D_{a} a_{x x}-k a b, & 0<x<\infty, t>0, \\ b_{t}=-k a b, & 0<x<\infty, t>0, \\ c_{t}=D c_{x x}+k a b-\lambda c \tilde{H}\left(\int_{0}^{t}\left(c(x, \tau)-C_{s}\right)^{+} d \tau\right), & 0<x<\infty, t>0, \\ a(0, t)=a_{0}>0, & t>0, \\ c_{x}(0, t)=0, & t>0, \\ a(x, 0)=c(x, 0)=0 ; \quad b(x, 0)=b_{0}>0, & 0<x<\infty .\end{cases}
$$

It is our aim to study Problem $\left(\mathrm{P}_{k}^{*}\right)$ for very large values of $k$, or in other words to study its asymptotic behavior as $k$ tends to infinity. With this purpose in mind, we shall use the notation $a_{k}, b_{k}, c_{k}$ for a solution of Problem $\left(\mathrm{P}_{k}^{*}\right)$. Our strategy is to first solve for $a_{k}$ and $b_{k}$, then compute $k a_{k} b_{k}$ and use this expression as a given source in the equation for $c_{k}$. In particular, we are interested in the validity of this approach as $k$ tends to infinity. The behavior of $a_{k}$ and $b_{k}$ has been studied in [1] and [2]. In particular, it has been shown that $a:=\lim _{k \rightarrow \infty} a_{k}$ is the solution of a one-phase Stefan problem with melting boundary $\zeta(t)=\alpha \sqrt{t}$ for some positive constant $\alpha$. Thus we are lead to study the problem

$$
\left(\mathrm{P}_{k}\right) \begin{cases}\left.c_{k t}=D c_{k x x}+k a_{k} b_{k}-\lambda c_{k} \tilde{H}\left(\int_{0}^{t}\left(c_{k}(x, \tau)-C_{s}\right)^{+}\right) d \tau\right), & 0<x<\infty, t>0, \\ c_{k x}(0, t)=0, & t>0, \\ c_{k}(x, 0)=0, & x>0,\end{cases}
$$

and in particular the singular limit of $c_{k}$ as $k \rightarrow \infty$. In order to do so, we first study the asymptotic behavior of the source term $k a_{k} b_{k}$. 
2.1 The Singular Limit of $k a_{k} b_{k}$ as $k \rightarrow \infty$

In this section we consider the problem,

$$
\left(\mathrm{S}_{k}\right) \begin{cases}a_{k t}=D_{a} a_{k x x}-k a_{k} b_{k}, & 0<x<\infty, t>0, \\ b_{k t}=-k a_{k} b_{k}, & 0<x<\infty, t>0, \\ a_{k}(0, t)=a_{0}>0, & t>0, \\ a_{k}(x, 0)=0, \quad b_{k}(x, 0)=b_{0}>0, & x>0 .\end{cases}
$$

It follows from the results of [1] and [2] that, as $k \rightarrow \infty$, the unique classical solution $\left(a_{k}, b_{k}\right)$ of system $\left(\mathrm{S}_{k}\right)$ is such that

$$
\left(a_{k}, b_{k}\right) \rightarrow(a, b) \quad \text { in } L_{\mathrm{loc}}^{p}\left(\mathbb{R}^{+} \times(0, T)\right) \text { and a.e. }
$$

for all $p \in[1, \infty)$, with $a b=0$, where $a$ is the unique solution of the classical one phase Stefan problem

$$
\begin{cases}a_{t}=D_{a} a_{x x}, & 0<x<\zeta(t), t>0, \\ a(0, t)=a_{0}, & t>0, \\ \zeta^{\prime}(t)=-\frac{D_{a}}{b_{0}} \frac{\partial a}{\partial x}\left(\zeta(t)^{-}, t\right), & t>0, \\ a(\zeta(t), t)=0, & t>0, \\ a(x, 0)=0, & x>0, \\ \zeta(0)=0 & \end{cases}
$$

and

$$
\begin{cases}b=0 & \text { in }\{0<x<\zeta(t), t>0\}, \\ b=b_{0} & \text { in }\{x>\zeta(t), t>0\} .\end{cases}
$$

Let us first recall a result of [2] which will be our starting point.

Theorem 2.1 For all $T>0$, it holds that

$$
\int_{0}^{T} \int_{\mathbb{R}^{+}} a_{k} b_{k} \leqq \frac{C}{k},
$$

where the constant $C$ depends on $T$.

Corollary 2.2 There exists a Radon measure $\mu$ such that as $k \rightarrow \infty$

$$
k a_{k} b_{k} \rightarrow \mu
$$

(along a subsequence) in the sense of weak convergence of measures.

Theorem 2.3 The measure $\mu$ is concentrated on the free boundary $\zeta$, or more precisely

$$
\mu=-b_{0} \frac{\partial}{\partial t} \tilde{H}(x-\zeta(t))=\zeta^{\prime}(t) b_{0} \delta(x-\zeta(t)),
$$

for all $x>0, t>0$. 
Proof Let $T>0$ be arbitrary. We multiply the equation for $b_{k}$ by a continuously differentiable test function with compact support in $(0, \infty) \times(0, T)$, namely $\phi \in C_{c}^{1}\left(\mathbb{R}^{+} \times(0, T)\right)$. This yields

$$
\int_{0}^{T} \int_{\mathbb{R}} b_{k t} \phi=-\int_{0}^{T} \int_{\mathbb{R}} k a_{k} b_{k} \phi
$$

for all $\phi \in C_{c}^{1}\left(\mathbb{R}^{+} \times(0, T)\right)$, which gives after integration by parts,

$$
\int_{0}^{T} \int_{\mathbb{R}}-b_{k} \phi_{t}+k a_{k} b_{k} \phi=0
$$

for all $\phi \in C_{c}^{1}\left(\mathbb{R}^{+} \times(0, T)\right)$. Letting $k \rightarrow \infty$ in the equation above, we obtain

$$
\int_{0}^{T} \int_{\mathbb{R}}-b \phi_{t}+\mu \phi=0
$$

for all $\phi \in C_{c}^{1}\left(\mathbb{R}^{+} \times(0, T)\right)$, in which we perform the substitution

$$
b=b_{0} \tilde{H}(x-\zeta(t)),
$$

which follows from (5), to obtain

$$
\int_{0}^{T} \int_{\mathbb{R}}\left(-b_{0} \tilde{H}(x-\zeta(t)) \phi_{t}+\mu \phi\right)=0,
$$

for all $\phi \in C_{c}^{1}\left(\mathbb{R}^{+} \times(0, T)\right)$, that is,

$$
\int_{0}^{T} \int_{\mathbb{R}}\left(b_{0} \frac{\partial}{\partial t} \tilde{H}(x-\zeta(t))+\mu\right) \phi=0,
$$

for all $\phi \in C_{c}^{1}\left(\mathbb{R}^{+} \times(0, T)\right)$. This completes the proof of Theorem 2.3.

It also follows from [1] that

$$
\zeta(t)=\alpha \sqrt{t}, \quad t>0
$$

for some positive constant $\alpha$. Therefore the measure $\mu$ is given by:

\section{Corollary 2.4}

$$
\mu=-b_{0} \frac{\partial}{\partial t} \tilde{H}(x-\alpha \sqrt{t})=\frac{b_{0} \alpha}{2 \sqrt{t}} \delta(x-\alpha \sqrt{t}) .
$$

The next step is to prove the existence of a solution $c_{k}$ of Problem $\left(\mathrm{P}_{k}\right)$ and to show that $c_{k} \rightarrow c$ along a subsequence as $k \rightarrow \infty$, where $c$ satisfies

(P) $\begin{cases}c_{t}=D c_{x x}+\frac{b_{0} \alpha}{2 \sqrt{t}} \delta(x-\alpha \sqrt{t})-\lambda c \tilde{H}\left(\int_{0}^{t}\left(c(x, \tau)-C_{s}\right)^{+} d \tau\right), & 0<x<\infty, t>0, \\ c_{x}(0, t)=0, & t>0, \\ c(x, 0)=0, & x>0\end{cases}$

in a weak sense, to be defined later. We begin with. 
2.2 The Limiting Behavior as $k \rightarrow \infty$ of the Solution of a Corresponding Linear Problem

In this section, we consider the linear problem

$$
\left(\mathrm{Q}_{k}\right) \quad \begin{cases}\psi_{t}=D \psi_{x x}+k a_{k} b_{k}, & 0<x<\infty, t>0, \\ \psi_{x}(0, t)=0, & t>0, \\ \psi(x, 0)=0, & x>0,\end{cases}
$$

and we prove the following result.

Theorem 2.5 As $k \rightarrow \infty$ the solution $\psi_{k}$ of Problem $\left(Q_{k}\right)$ converges to the unique weak solution $\psi$ of the problem

$$
\text { (Q) } \begin{cases}\psi_{t}=D \psi_{x x}+\frac{b_{0} \alpha}{2 \sqrt{t}} \delta(x-\alpha \sqrt{t}), & 0<x<\infty, t>0, \\ \psi_{x}(0, t)=0, & t>0, \\ \psi(x, 0)=0, & x>0 .\end{cases}
$$

Proof We define the new unknown function

$$
z_{k}(x, t)=\int_{0}^{t} \psi_{k}(x, \tau) d \tau,
$$

where $\psi_{k}$ is the unique classical solution of Problem $\left(\mathrm{Q}_{k}\right)$. Then $z_{k}$ is the solution of the problem

$$
\begin{cases}z_{t}=D z_{x x}+\int_{0}^{t} k a_{k} b_{k}, & 0<x<\infty, t>0 \\ z_{x}(0, t)=0, & t>0, \\ z(x, 0)=0, & x>0 .\end{cases}
$$

Since $b_{k t}=-k a_{k} b_{k}$, it follows that

$$
0 \leqq \int_{0}^{t} k a_{k} b_{k}=b_{0}-b_{k}(t) \leqq b_{0},
$$

so that $\left\|\int_{0}^{t} k a_{k} b_{k}\right\|_{L^{\infty}((0, \infty) \times(0, T))} \leqq b_{0}$ for all $T>0$. We deduce that for all $p \in(1, \infty)$ and for all $R>0$ there exists a positive constant $C=C(R, p)$ such that

$$
\left\|z_{k}\right\|_{W_{p}^{2,1}((0, R) \times(0, T))} \leqq C,
$$

and that

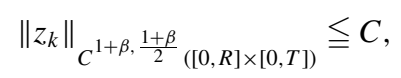

for all $\beta \in(0,1)$, where we have used the embedding

$$
W_{p}^{2,1}((0, R) \times(0, T)) \subset C^{1+\beta, \frac{1+\beta}{2}}([0, R] \times[0, T]),
$$

for all $p>3$, where $\beta=1-\frac{3}{p}$. Therefore as $k \rightarrow \infty$,

$$
z_{k} \rightarrow z, \quad \text { weakly in } W_{p}^{2,1}((0, R) \times(0, T)),
$$


and

$$
z_{k} \rightarrow z, \quad \text { in } C^{1+\beta, \frac{1+\beta}{2}}([0, R] \times[0, T]),
$$

for all $p \in(1, \infty)$ and $\beta \in(0,1)$, where $z$ is the unique solution of the problem

$$
\begin{cases}z_{t}=D z_{x x}+b_{0}-b_{0} \tilde{H}(x-\alpha \sqrt{t}), & 0<x<\infty, t>0, \\ z_{x}(0, t)=0, & t>0, \\ z(x, 0)=0, & x>0 .\end{cases}
$$

Taking the difference of the equations for $z_{k}$ and for $z$ we deduce that

$$
\begin{cases}\left(z_{k}-z\right)_{t}=D\left(z_{k}-z\right)_{x x}-\left(b_{k}-b\right), & 0<x<\infty, t>0, \\ \left(z_{k}-z\right)_{x}(0, t)=0, & t>0, \\ \left(z_{k}-z\right)(x, 0)=0, & x>0 .\end{cases}
$$

Therefore

$$
\left\|z_{k}-z\right\|_{W_{p}^{2,1}((0, R) \times(0, T))} \leqq C\left\|b_{k}-b\right\|_{L^{p}((0, R) \times(0, T))} .
$$

Thus, as $k \rightarrow \infty$,

$$
z_{k} \rightarrow z \quad \text { strongly in } W_{p}^{2,1}((0, R) \times(0, T)),
$$

for all $p \in(1, \infty)$ and in particular

$$
z_{k t} \rightarrow z_{t} \quad \text { strongly in } L^{p}((0, R) \times(0, T)),
$$

so that, if we define $\psi=z_{t}$,

$$
\psi_{k} \rightarrow \psi \quad \text { strongly in } L^{p}((0, R) \times(0, T)),
$$

as $k \rightarrow \infty$, where $\psi$ is the weak solution of the problem

$$
\begin{cases}\psi_{t}=D \psi_{x x}+\frac{b_{0} \alpha}{2 \sqrt{t}} \delta(x-\alpha \sqrt{t}), & 0<x<\infty, t>0, \\ \psi_{x}(0, t)=0, & t>0, \\ \psi(x, 0)=0, & x>0 .\end{cases}
$$

We present below a complete characterization of the function $\psi$. We set

$$
\psi(x, t)=\Psi(\eta) \quad \text { with } \eta=\frac{x}{\sqrt{t}} .
$$

Then $\Psi$ satisfies the problem

$$
\left\{\begin{array}{l}
D \Psi^{\prime \prime}+\frac{\eta}{2} \Psi^{\prime}+\frac{b_{0} \alpha}{2} \delta(\eta-\alpha)=0, \quad \eta \in(0, \infty), \\
\Psi_{\eta}(0)=0 \\
\Psi(\infty)=0
\end{array}\right.
$$

so that

$$
\Psi(\eta)= \begin{cases}A\left(D, b_{0}, \alpha\right):=\left(b_{0} \alpha e^{\frac{\alpha^{2}}{4 D}} /(2 D)\right) \int_{\alpha}^{\infty} e^{-\frac{s^{2}}{4 D},}, & \text { if } \eta \leqq \alpha, \\ \left(b_{0} \alpha e^{\frac{\alpha^{2}}{4 D}} /(2 D)\right) \int_{\eta}^{\infty} e^{-\frac{s^{2}}{4 D}}, & \text { if } \eta>\alpha,\end{cases}
$$

see Fig. 6. 
Fig. 6 Shape of the function $\Psi$

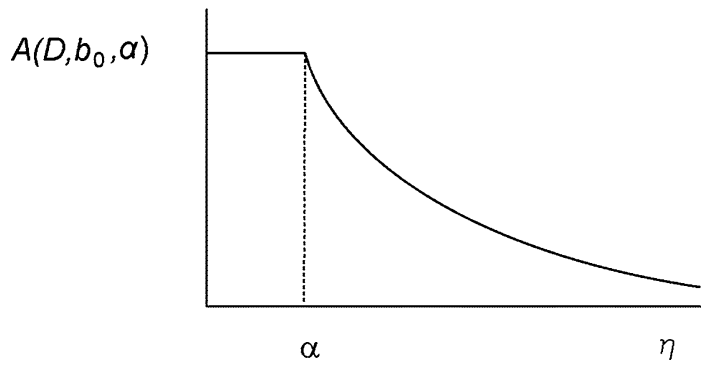

In particular

$$
\Psi \in B C\left(\mathbb{R}^{+}\right), \quad \lim _{\eta \rightarrow \infty} \Psi(\eta)=0,
$$

and thus

$$
\psi \in B C\left(\mathbb{R}^{+} \times \mathbb{R}^{+}\right), \quad \lim _{x \rightarrow \infty} \psi(x, t)=0 \quad \text { for } t \in \mathbb{R}^{+}, \quad \psi(x, 0)=0 \quad \text { for } x \in \mathbb{R}^{+} .
$$

More precisely, the following results hold.

Lemma $2.6 \psi \in L^{2}\left(0, T ; H^{1}(0, \infty)\right) \cap H^{1}\left((0, \infty) \times\left(\tau_{0}, T\right)\right)$ for any $0<\tau_{0}<T$.

Proof Recall that $\psi(x, t)=\Psi\left(\frac{x}{\sqrt{t}}\right)$ so that

$$
\Psi^{\prime}(\eta) \equiv 0 \quad \text { when } \eta<\alpha ; \quad \Psi^{\prime}(\eta)=-\frac{b_{0} \alpha}{2 D} e^{\frac{\alpha^{2}-\eta^{2}}{4 D}} \quad \text { when } \eta>\alpha .
$$

We only need to prove that $\psi_{t} \in L^{2}\left((0, \infty) \times\left(\tau_{0}, T\right)\right)$ for any $0<\tau_{0}<T$. To that purpose, we prove that there exists a constant $C_{1}>0$ such that, for every $t^{*}>0$,

$$
\int_{t^{*}}^{\infty} \int_{0}^{\infty} \psi_{t}^{2} d x d t \leq \frac{C_{1}}{\sqrt{t^{*}}}
$$

Indeed, $\psi_{t}=-\frac{\eta}{2 t} \Psi^{\prime}(\eta)$. For fixed $t$, we have that $d x=\sqrt{t} d \eta$ and

$$
\int_{t^{*}}^{\infty} \int_{0}^{\infty} \psi_{t}^{2} d x d t=\int_{t^{*}}^{\infty} \int_{0}^{\infty} \frac{\left(\eta \Psi^{\prime}(\eta)\right)^{2}}{4 t^{\frac{3}{2}}} d \eta d t
$$

The proof now follows from (6).

\subsection{Existence of a Solution of Problem $\left(\mathrm{P}_{k}\right)$}

We extend the unknown concentration $c_{k}$ to the whole domain $\mathbb{R} \times \mathbb{R}^{+}$according to

$$
c_{k}(-x, t)=c_{k}(x, t), \quad x>0, t>0 .
$$

and $a_{k}$ and $b_{k}$ similarly. Problem $\left(\mathrm{P}_{k}\right)$ becomes

$$
\left(\mathrm{P}_{k}\right) \begin{cases}c_{t}=D c_{x x}+k a_{k} b_{k}-\lambda c \tilde{H}\left(\int_{0}^{t}\left(c-C_{s}\right)^{+}\right), & x \in \mathbb{R}, t>0, \\ c(x, 0)=0, & x \in \mathbb{R} .\end{cases}
$$


In order to be able to work with partial differential equations with a bounded right-hand side, we perform a change of the unknown function. We set

$$
\psi_{k}(-x, t)=\psi_{k}(x, t), \quad x>0, t>0,
$$

and

$$
\tilde{c}_{k}=c_{k}-\psi_{k}
$$

Problem $\left(\mathrm{P}_{k}\right)$ can be rewritten as

$$
\left(\mathrm{P}_{k}\right) \begin{cases}\tilde{c}_{t}=D \tilde{c}_{x x}-\lambda\left(\tilde{c}+\psi_{k}\right) \tilde{H}\left(\int_{0}^{t}\left(\tilde{c}+\psi_{k}-C_{s}\right)^{+}\right), & x \in \mathbb{R}, t>0, \\ \tilde{c}(x, 0)=0, & x \in \mathbb{R} .\end{cases}
$$

However we will only prove the existence of a weak solution of a slightly different problem, namely Problem $\left(\mathcal{P}_{k}\right)$, which one obtains by replacing the Heaviside function $\tilde{H}$ in Problem $\left(\mathrm{P}_{k}\right)$ by the Heaviside graph

$$
H(y) \in \begin{cases}0 & \text { when } y<0 \\ {[0,1]} & \text { when } y=0 \\ 1 & \text { when } y>0\end{cases}
$$

Definition A weak solution of Problem $\left(\mathcal{P}_{k}\right)$ is a function pair $\left\{c_{k}, \mathcal{X}_{k}\right\}$ with the properties:

(i) for each $T>0, c_{k}-\psi_{k} \in C^{1+\gamma, \frac{1+\gamma}{2}}(\mathbb{R} \times[0, T]) \cap H_{l o c}^{1}(\mathbb{R} \times[0, T])$ for all $0<\gamma<1$;

(ii) for all $\varphi \in C^{1}(\mathbb{R} \times[0, T])$ such that $\varphi$ vanishes for large $|x|$ and for $t=T$,

$$
\int_{0}^{T} \int_{\mathbb{R}}\left(\psi_{k}-c_{k}\right) \varphi_{t}=\int_{0}^{T} \int_{\mathbb{R}}\left[D\left(\psi_{k}-c_{k}\right)_{x} \varphi_{x}-\lambda c_{k} \varphi \mathcal{X}_{k}\right],
$$

where $\mathcal{X}_{k} \in H\left(\int_{0}^{t}\left(c_{k}-C_{s}\right)^{+}(x, \tau) d \tau\right)$ is such that $\mathcal{X}_{k}(x, t)=0$ if $c_{k}(x, \tau)-C_{s}<0$ for all $\tau \leq t$.

The remainder of this section is devoted to proving existence of a weak solution of Problem $\left(\mathcal{P}_{k}\right)$. To begin with we consider the boundary value problem

$$
\begin{cases}\psi_{t}=D \psi_{x x}+k a_{k} b_{k}, & x \in(-R, R), t>0 \\ \psi( \pm R, t)=0, & t>0 \\ \psi(x, 0)=0, & x \in(-R, R)\end{cases}
$$

and denote its unique solution by $\psi_{k_{R}}$. Next, we prove the existence of a solution of the problem

$$
\left(\mathrm{P}_{k_{R}}\right) \begin{cases}\tilde{c}_{t}=D \tilde{c}_{x x}-\lambda\left(\tilde{c}+\psi_{k_{R}}\right) \mathcal{H}_{\frac{1}{R}}\left(\int_{0}^{t}\left(\tilde{c}+\psi_{k_{R}}-C_{s}\right)^{+}\right), & x \in(-R, R), t>0, \\ \tilde{c}( \pm R, t)=0, & t>0, \\ \tilde{c}(x, 0)=0, & x \in(-R, R),\end{cases}
$$


where $\mathcal{H}_{\varepsilon}$ is a smooth nondecreasing approximation of the Heaviside graph $H$ such that

$$
\mathcal{H}_{\varepsilon}(s)=H(s) \quad \text { for all } s>\varepsilon \text { and } s<0 .
$$

We consider the map $\Gamma: \phi \rightarrow \gamma$, where $\gamma$ is the unique solution of the auxiliary problem

$$
\left(\mathrm{P}_{A}\right) \begin{cases}\gamma_{t}=D \gamma_{x x}-\lambda\left(\gamma+\psi_{k_{R}}\right) \mathcal{H}_{\frac{1}{R}}\left(\int_{0}^{t}\left(\phi+\psi_{k_{R}}-C_{s}\right)^{+}\right), & x \in(-R, R), t>0, \\ \gamma( \pm R, t)=0, & t>0, \\ \gamma(x, 0)=0, & x \in(-R, R) .\end{cases}
$$

We set

$$
\mathcal{L} u=u_{t}-D u_{x x}+\lambda\left(u+\psi_{k_{R}}\right) \mathcal{H}_{\frac{1}{R}}\left(\int_{0}^{t}\left(\phi+\psi_{k_{R}}-C_{s}\right)^{+}\right),
$$

and observe that

$$
\mathcal{L} 0=\lambda \psi_{k_{R}} \mathcal{H}_{\frac{1}{R}}\left(\int_{0}^{t}\left(\phi+\psi_{k_{R}}-C_{s}\right)^{+}\right) \geqq 0
$$

and that

$$
\mathcal{L}\left(-\psi_{k_{R}}\right)=\left(-\psi_{k_{R}}\right)_{t}-D\left(-\psi_{k_{R}}\right)_{x x}=-k a_{k} b_{k} \leqq 0 .
$$

We define

$$
\begin{aligned}
& \mathcal{C}=\left\{u \in C([-R, R] \times[0, T]),-\psi_{k_{R}} \leqq u \leqq 0,\right. \\
&u(-x, t)=u(x, t) \text { for all }(x, t) \in(-R, R) \times(0, T)\},
\end{aligned}
$$

and remark that $\Gamma$ maps $\mathcal{C}$ into itself, which implies that

$$
\left|\gamma_{t}-D \gamma_{x x}\right| \leqq \lambda\left\|\psi_{k_{R}}\right\|_{L^{\infty}((-R, R) \times(0, T))} \leqq C_{k} .
$$

Therefore there exists a positive constant $\tilde{C}_{k}$ such that

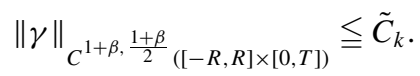

Thus $\Gamma$ is a compact map from $\mathcal{C}$ into itself. Furthermore it is continuous; indeed suppose that

$$
\phi_{n} \rightarrow \phi \quad \text { in } C([-R, R] \times[0, T]), \quad \text { as } n \rightarrow \infty,
$$

then as $n \rightarrow \infty$

$$
\mathcal{F}_{n}=\mathcal{H}_{\frac{1}{R}}\left(\int_{0}^{t}\left(\phi_{n}+\psi-C_{s}\right)^{+}\right)
$$

converges uniformly to its limit

$$
\mathcal{F}=\mathcal{H}_{\frac{1}{R}}\left(\int_{0}^{t}\left(\phi+\psi-C_{s}\right)^{+}\right)
$$

in $[-R, R] \times[0, T]$. Next, we define $\gamma_{n}=\Gamma\left(\phi_{n}\right)$ and take the difference of the equations for $\gamma$ and for $\gamma_{n}$ to obtain

$$
\left(\gamma-\gamma_{n}\right)_{t}=D\left(\gamma-\gamma_{n}\right)_{x x}-\lambda\left(\gamma-\gamma_{n}\right) \mathcal{F}-\lambda \gamma_{n}\left(\mathcal{F}-\mathcal{F}_{n}\right)+\lambda \psi_{k_{R}}\left(\mathcal{F}_{n}-\mathcal{F}\right) .
$$


Since $\left\|\gamma_{n}\left(\mathcal{F}_{n}-\mathcal{F}\right)\right\|_{C([-R, R] \times[0, T])}$ and $\left\|\psi_{k_{R}}\left(\mathcal{F}_{n}-\mathcal{F}\right)\right\|_{C([-R, R] \times[0, T])}$ tend to zero as $n \rightarrow \infty$, it follows, since also $\gamma$ and $\gamma_{n}$ satisfy the same boundary and initial conditions, that

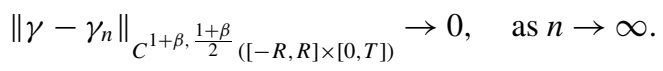

Therefore it follows from the Schauder fixed point theorem that the map $\Gamma$ has a fixed point $\tilde{c}_{k_{R}}$ which is a classical solution of Problem $\left(\mathrm{P}_{k_{R}}\right)$. Since $\left\|k a_{k} b_{k}\right\|_{L^{\infty}(\mathbb{R} \times(0, T))} \leqq k a_{0} b_{0}$, it follows that

$$
\left\|\psi_{k_{R}}\right\|_{C^{1+\beta, \frac{1+\beta}{2}}([-R, R] \times[0, T])} \leqq C_{k}
$$

for all $\beta \in(0,1)$; in turn since

$$
-\psi_{k_{R}} \leqq \tilde{c}_{k_{R}} \leqq 0,
$$

we deduce that

$$
\left\|\left(\tilde{c}_{k_{R}}+\psi_{k_{R}}\right) \mathcal{H}_{\frac{1}{R}}\left(\int_{0}^{t}\left(\tilde{c}_{k_{R}}+\psi_{k_{R}}-C_{s}\right)^{+}\right)\right\|_{L^{\infty}((-R, R) \times(0, T))} \leqq C_{k} .
$$

Therefore there exist $\tilde{c}_{k_{R_{n}}}$ and $\tilde{c}_{k}$ such that

$$
\tilde{c}_{k_{R_{n}}} \rightarrow \tilde{c}_{k} \quad \text { as } R_{n} \rightarrow \infty
$$

in $C^{1+\beta, \frac{1+\beta}{2}}([-L, L] \times[0, T])$ for all $L>0$. Then

$$
\mathcal{E}_{k_{R_{n}}}=\int_{0}^{t}\left(\tilde{c}_{k_{R_{n}}}+\psi_{k_{R_{n}}}-C_{s}\right)^{+} \rightarrow \mathcal{E}_{k}=\int_{0}^{t}\left(\tilde{c}_{k}+\psi_{k}-C_{s}\right)^{+},
$$

as $R_{n} \rightarrow \infty$, uniformly in compact sets of $\mathbb{R} \times[0, T]$, and there exist $\mathcal{X}_{k} \in H\left(\mathcal{E}_{k}\right)$ and a subsequence of $\mathcal{E}_{k_{R_{n}}}$ which we denote again by $\mathcal{E}_{k_{R_{n}}}$ such that

$$
\mathcal{H}_{\frac{1}{R_{n}}}\left(\mathcal{E}_{k_{R_{n}}}\right) \rightarrow \mathcal{X}_{k}
$$

as $R_{n} \rightarrow \infty$, weakly in $L^{2}((-L, L) \times(0, T))$ for all $L>0$. Next we show that the function pair $\left\{\tilde{c}_{k}, \mathcal{X}_{k}\right\}$ is a weak solution of the Problem $\left(\mathcal{P}_{k}\right)$.

Indeed extend $\tilde{c}_{k_{R_{n}}}$ by zero for $|x| \geqq R_{n}$. Then $\tilde{c}_{k_{R_{n}}}$ satisfies the integral identity

$$
\int_{0}^{T} \int_{\mathbb{R}}\left\{\tilde{c}_{k_{R_{n}}} \sigma_{t}+D \tilde{c}_{k_{R_{n}}} \sigma_{x}-\lambda\left(\tilde{c}_{k_{R_{n}}}+\psi_{k_{R_{n}}}\right) \mathcal{H}_{\frac{1}{R_{n}}}\left(\mathcal{E}_{k_{R_{n}}}\right) \sigma\right\}=0,
$$

for all $\sigma \in C^{2,1}(\mathbb{R} \times[0, T])$ such that $\sigma$ vanishes for large $|x|$ and for $t=T$. Letting $R_{n} \rightarrow \infty$, we deduce that

$$
\int_{0}^{T} \int_{\mathbb{R}}\left\{\tilde{c}_{k} \sigma_{t}+D \tilde{c}_{k_{x}} \sigma_{x}-\lambda\left(\tilde{c}_{k}+\psi_{k}\right) \mathcal{X}_{k} \sigma\right\}=0,
$$

for all test functions $\sigma \in C^{2,1}(\mathbb{R} \times[0, T])$ with the above properties.

We claim that $\mathcal{X}_{k}(x, t)=1$ if $\mathcal{E}_{k}(x, t)>0, \mathcal{X}_{k}(x, t)=0$ if $\tilde{c}_{k}(x, \tau)+\psi_{k}(x, \tau)$ $-C_{s}<0$ for all $\tau \leq t$ and that $\mathcal{X}_{k}(x, t) \in[0,1]$ anywhere. To see this, we discuss the case that $\mathcal{E}_{k}(x, t)>0$, the other cases being similar. If we choose $n$ so large that
(i) $\frac{1}{R_{n}}<\frac{\mathcal{E}_{k}(x, t)}{2}$
(ii) $\mathcal{E}_{k_{R_{n}}}(x, t)>\frac{\mathcal{E}_{k}(x, t)}{2}$, 
then, by $(9), \mathcal{H}_{\frac{1}{R_{n}}}\left(\mathcal{E}_{k_{R_{n}}(x, t)}\right)=1$. This implies that $\mathcal{X}_{k}(x, t)=1$ as well.

Therefore we have proved the existence of a weak solution $\left\{\tilde{c}_{k}, \mathcal{X}_{k}\right\}$ of Problem $\left(\mathcal{P}_{k}\right)$ such that

$$
-\psi_{k} \leqq \tilde{c}_{k} \leqq 0 \quad \text { and } \quad \tilde{c}_{k}(-x, t)=\tilde{c}_{k}(x, t) \quad \text { for all }(x, t) \in \mathbb{R} \times[0, T),
$$

where we recall that

$$
\left\|\psi_{k}\right\|_{L^{p}((-R, R) \times(0, T))} \leqq C(R, p)
$$

for all $p \in[1, \infty)$ and $R>0$.

2.4 Singular Limit as $k \rightarrow \infty$ of Solutions of Problem $\left(\mathcal{P}_{k}\right)$

We consider the limit Problem $(\hat{\mathcal{P}})$ which we define by

$$
(\hat{\mathcal{P}}) \quad \begin{cases}c_{t}=D c_{x x}+\frac{b_{0} \alpha}{2 \sqrt{t}} \delta(x-\alpha \sqrt{t})-\lambda c \mathcal{X}(x, t), & 0<x<\infty, t>0, \\ c_{x}(0, t)=0, & t>0, \\ c(x, 0)=0, & x>0,\end{cases}
$$

where

$$
\mathcal{X}(x, t) \in H\left(\int_{0}^{t}\left(c-C_{s}\right)^{+}(x, \tau) d \tau\right)
$$

is such that $\mathcal{X}(x, t)=0$ when $c(x, \tau)-C_{s}<0$ for all $\tau \leq t$.

Definition A weak solution of Problem $(\hat{\mathcal{P}})$ is a function pair $\{c, \mathcal{X}\}$ with the properties:

(1) for each $T>0, c-\psi \in C^{1+\gamma, \frac{1+\gamma}{2}}([0, \infty) \times[0, T]) \cap H_{l o c}^{1}([0, \infty) \times[0, T])$ for all $0<\gamma<1$

(2) for all $\varphi \in C^{1}([0, \infty) \times[0, T])$ such that $\varphi$ vanishes for large $|x|$ and for $t=T$,

$$
\int_{0}^{T} \int_{0}^{\infty}(\psi-c) \varphi_{t} d x d t=\int_{0}^{T} \int_{0}^{\infty}\left[D(\psi-c)_{x} \varphi_{x}-\lambda c \varphi \mathcal{X}\right] d x d t
$$

where $\mathcal{X} \in H\left(\int_{0}^{t}\left(c-C_{s}\right)^{+}(x, \tau) d \tau\right)$ is such that $\mathcal{X}(x, t)=0$ when $c(x, \tau)-C_{s}<0$ for all $\tau \leq t$.

We remark that by Lemma 3.2 below a weak solution $c$ of Problem $(\hat{\mathcal{P}})$ also belongs to $L^{2}\left(0, T ; H^{1}(0, \infty)\right)$.

Again restricting the functions $c_{k}, \tilde{c}_{k}$ and $\psi_{k}$ to $[0, \infty) \times[0, T]$, we prove the following result.

Theorem 2.7 There exists subsequences $\left\{c_{k n}\right\}$ and $\left\{\mathcal{X}_{k n}\right\}$, and functions

$$
\begin{aligned}
& c \in C^{1+\gamma, \frac{1+\gamma}{2}}([0, R] \times[0, T]) \quad \text { for all } \gamma \in(0,1) \text { and } \\
& \mathcal{X} \in L^{2}\left(((0, L) \times(0, T)), \quad \text { such that, as } \quad k_{n} \rightarrow \infty, \quad c_{k n}-\psi_{k n} \rightarrow c-\psi\right.
\end{aligned}
$$

in $C^{1+\gamma, \frac{1+\gamma}{2}}([0, R] \times[0, T])$ for all $\gamma \in(0,1)$ and $\mathcal{X}_{k n} \rightarrow \mathcal{X}$ weakly in $L^{2}((0, L) \times(0, T))$, $R$ and $T$ positive. The function $\mathcal{X}$ satisfies $(11)$, the pair $(c, \mathcal{X})$ is a weak solution of Problem $(\hat{\mathrm{P}})$, and the function $c$ is such that $0 \leq c \leq \psi$. 
Proof In what follows we use the notation

$$
\mathcal{F}_{k}=\left(\tilde{c}_{k}+\psi_{k}\right) \mathcal{X}_{k}
$$

and deduce from the inequalities $0 \leqq \mathcal{F}_{k} \leqq \psi_{k}$ that

$$
\left\|\mathcal{F}_{k}\right\|_{L^{p}((0, R) \times(0, T))} \leqq C(R, p) .
$$

Therefore, we have that

$$
\left\|\tilde{c}_{k}\right\|_{W_{p}^{2,1}((0, R) \times(0, T))} \leqq C(R, p)
$$

for all $R>0$ and $p \in[0, \infty)$, which implies that

$$
\left\|\tilde{c}_{k}\right\|_{C^{1+\beta, \frac{1+\beta}{2}}([0, \infty) \times[0, T])} \leqq C(R, \beta)
$$

for all $\beta \in(0,1)$. As a consequence there exist a function $\tilde{c} \in C^{1+\beta, \frac{1+\beta}{2}}([0, \infty) \times(0, T)) \cap$ $W_{p, \text { loc }}^{2,1}((0, R) \times[0, T])$, and a subsequence $\left\{\tilde{c}_{k_{n}}\right\}$ such that as $k_{n} \rightarrow \infty$

$$
\tilde{c}_{k_{n}} \rightarrow \tilde{c} \quad \text { in } C^{1+\beta, \frac{1+\beta}{2}}([0, R] \times[0, T])
$$

for all $\beta \in(0,1)$ and

$$
\tilde{c}_{k_{n}} \rightarrow \tilde{c} \quad \text { weakly in } W_{p}^{2,1}((0, R) \times(0, T)) \text { for all } R>0 \text { and } p \in[1, \infty) .
$$

Furthermore there exists a function $\mathcal{X} \in[0,1]$ and a subsequence of $\mathcal{X}_{k_{n}}$ which we denote again by $\mathcal{X}_{k_{n}}$ such that as $k_{n} \rightarrow \infty$,

$$
\mathcal{X}_{k_{n}} \rightarrow \mathcal{X} \quad \text { weakly in } L^{2}((0, L) \times(0, T))
$$

for each $L>0$. If $(x, t)$ is such that

$$
\int_{0}^{t}\left(\tilde{c}+\psi-C_{s}\right)^{+}(x, \tau) d \tau>0,
$$

then there exists $\kappa>0$ such that

$$
\int_{0}^{t}\left(\tilde{c}_{k_{n}}+\psi_{k_{n}}-C_{s}\right)^{+}(x, \tau) d \tau>0
$$

for all $k_{n} \geqq \kappa$, so that

$$
\mathcal{X}_{k_{n}}(x, t)=1, \quad \text { for all } k_{n} \geqq \kappa,
$$

and therefore

$$
\mathcal{X}(x, t)=1 \text {. }
$$

If $(x, t)$ is such that

$$
\tilde{c}(x, \tau)+\psi(x, \tau)-C_{s}<0 \quad \text { for all } \tau \leq t
$$

we have that

$$
\mathcal{X}(x, t)=0 .
$$


The argument is similar to the previous one: obviously, there is a $\delta>0$ such that $\tilde{c}(x, \tau)+$ $\psi(x, \tau)-C_{s} \leq-\delta$ for all $\tau \leq t$. Therefore, if $n$ is sufficiently large, $\tilde{c}_{k_{n}}(x, \tau)+\psi_{k_{n}}(x, \tau)-$ $C_{s}<-\frac{\delta}{2}$ for all $\tau \leq t$. This, in turn, implies that $\mathcal{X}_{k_{n}}(x, t)=0$. Taking the limit, we see that $\mathcal{X}(x, t)=0$ as well. Obviously, in the remaining cases it holds that

$$
\mathcal{X} \in[0,1] .
$$

Now letting $k \rightarrow \infty$ in (10) we obtain the integral equality

$$
\int_{0}^{T} \int_{0}^{\infty}\left\{\tilde{c} \sigma_{t}+D \tilde{c}_{x} \sigma_{x}-\lambda(\tilde{c}+\psi) \mathcal{X} \sigma\right\}=0,
$$

for all $\sigma \in C^{2,1}([0, \infty) \times[0, T))$ such that $\sigma$ vanishes for large $|x|$ and for $t=T$. Here,

$$
\mathcal{X} \in H\left(\int_{0}^{t}\left(\tilde{c}+\psi-C_{s}\right)^{+}\right) .
$$

Therefore the function pair $\{\tilde{c}, \mathcal{X}\}$ is a weak solution of the problem

$$
(\mathcal{P}) \quad \begin{cases}\tilde{c}_{t}=D \tilde{c}_{x x}-\lambda(\tilde{c}+\psi) \mathcal{X}, & x \in(0, \infty), t>0, \\ \tilde{c}_{x}(0, t)=0, & t>0, \\ \tilde{c}(x, 0)=0, & x>0 .\end{cases}
$$

Writing $c=\tilde{c}+\psi$ we have that $0 \leq c \leq \psi$. The function pair $\{c, \mathcal{X}\}$ is a weak solution of Problem $(\hat{\mathcal{P}})$.

Finally, we mention a useful scaling property of Problem $(\hat{\mathcal{P}})$ :

Lemma 2.8 Define, for $\beta>0, u_{\beta}(x, t)=c(x \sqrt{\beta}, t \beta)$. Then $u_{\beta}$ satisfies

$$
\begin{cases}u_{\beta t}=D u_{\beta x x}+\frac{b_{0} \alpha}{2 \sqrt{t}} \delta(x-\alpha \sqrt{t})-\beta \lambda u_{\beta} \mathcal{H}_{\beta}(x, t), & 0<x<\infty, t>0 \\ u_{\beta x}(0, t)=0, & t>0, \\ u_{\beta}(x, 0)=0, & x>0,\end{cases}
$$

where

$$
\mathcal{H}_{\beta}(x, t) \in H\left(\int_{0}^{t}\left(u_{\beta}(x, \tau)-C_{s}\right)^{+} d \tau\right)
$$

is such that $\mathcal{H}(x, t)=0$ when $u_{\beta}(x, \tau)-C_{s}<0$ for all $\tau \leq t$.

The proof is by direct computation. This lemma enables us to assume that $\lambda=1$, which we shall do from now on.

\section{Liesegang Bands}

Definition A Liesegang band is a connected component of the set

$$
\left\{(x, t) \in[0, \infty) \times[0, T): w(x, t):=\int_{0}^{t}\left(c(x, \tau)-C_{s}\right)^{+} d \tau>0\right\} .
$$


In this section, we shall prove that Liesegang bands do indeed exist under suitable conditions and that their starting points satisfy the time law that has been mentioned in Sect. 1 . Further it follows from the facts:

1. $c \leq \psi \leq A\left(D, b_{0}, \alpha\right)$, and

2. Liesegang bands can germinate only if $c>C_{s}$,

that Liesegang bands can only exist if the condition

$$
C_{s}<A\left(D, b_{0}, \alpha\right)
$$

is satisfied, which we shall assume from now on. Concerning the value of $A\left(D, b_{0}, \alpha\right)$, we have:

Lemma 3.1 $A\left(D, b_{0}, \alpha\right)$ only depends on $\frac{\alpha}{\sqrt{D}}$ and $b_{0}$. It is strictly increasing in $\frac{\alpha}{\sqrt{D}}$ and

$$
\lim _{\frac{\alpha}{\sqrt{D}} \rightarrow \infty} A\left(D, b_{0}, \alpha\right)=b_{0}
$$

Proof Define $\theta(D, \alpha):=\frac{\alpha e^{\frac{\alpha^{2}}{4 D}}}{D} \int_{\alpha}^{\infty} e^{\frac{-s^{2}}{4 D}} d s$. It is simple to see that $\theta(D, \alpha)=\tilde{\theta}(\zeta)$, with $\zeta=$ $\frac{\alpha}{\sqrt{D}}$ and that $\tilde{\theta}(0)=0$ and $\lim _{\zeta \rightarrow \infty} \theta(\zeta)=2$. Note that $\tilde{\theta}$ satisfies the differential equation

$$
\tilde{\theta}^{\prime \prime}=\tilde{\theta}+\frac{\zeta}{2} \tilde{\theta}^{\prime}-2
$$

It follows easily that $\tilde{\theta}$ cannot assume a maximum $>2$; nor can it assume a minimum or a saddle value $<2$. Finally, given that $\tilde{\theta}(0)=0$, by uniqueness it cannot happen that $\tilde{\theta}\left(\zeta^{*}\right)=2, \tilde{\theta}^{\prime}\left(\zeta^{*}\right)=0$ for any $\zeta^{*}<\infty$.

In the sequel, we shall need:

Lemma 3.2 Let c be a weak solution of Problem $(\hat{\mathcal{P}})$. Then

$$
\frac{1}{2} \int_{0}^{\infty} c(T)^{2} d x+\int_{0}^{T} \int_{0}^{\infty} D c_{x}^{2} d x d t+\int_{0}^{T} \int_{0}^{\infty} c^{2} \chi d x d t \leq b_{0} \alpha A\left(D, b_{0}, \alpha\right) \sqrt{T}
$$

Proof We know that $c$ is a weak solution of the equation

$$
c_{t}=D c_{x x}+\frac{b_{0} \alpha}{2 \sqrt{t}} \delta(x-\alpha \sqrt{t})-c \chi
$$

It follows from (7) and (14) that $c_{t} \in L^{2}\left((0, R) \times\left(t^{*}, T\right)\right)$ for all $R>0$ and $t^{*} \in(0, T]$. Given some $R^{*}>0$, let, for $R>R^{*}, \zeta_{R} \in C^{\infty}([0, \infty))$ be a smooth function such that

$$
\zeta_{R}(x) \in \begin{cases}1 & \text { when } 0 \leq x \leq R \\ {[0,1]} & \text { when } R \leq x \leq 2 R \\ 0 & \text { when } x \geq 2 R\end{cases}
$$


and such that $\left|\zeta_{R}^{\prime \prime}\right| \leq C$ for some positive constant $C$ which does not depend on $R$. We multiply (17) by $c \zeta$ and integrate by parts to deduce that for all $t^{*} \in(0, T]$

$$
\begin{aligned}
& \frac{1}{2} \int_{0}^{\infty}\left\{c(x, T)^{2}-c\left(x, t^{*}\right)^{2}\right\} \zeta_{R}(x) d x+D \int_{t^{*}}^{T} \int_{0}^{\infty} c_{x}^{2} \zeta_{R} d x d t+D \int_{t^{*}}^{T} \int_{R}^{2 R}\left\{\frac{c^{2}}{2}\right\}_{x} \zeta_{R}^{\prime} d x d t \\
& \quad+\int_{t^{*}}^{T} \int_{0}^{\infty} c^{2} \chi \zeta_{R} d x d t \leq b_{0} \alpha A\left(D, b_{0}, \alpha\right) \sqrt{T}
\end{aligned}
$$

in which we let $t^{*}$ tend to zero to obtain

$$
\begin{aligned}
& \frac{1}{2} \int_{0}^{\infty} c(x, T)^{2} \zeta_{R}(x, T) d x+D \int_{0}^{T} \int_{0}^{\infty} c_{x}^{2} \zeta_{R} d x d t+D \int_{0}^{T} \int_{R}^{2 R}\left\{\frac{c^{2}}{2}\right\}_{x} \zeta_{R}^{\prime} d x d t \\
& \quad+\int_{0}^{T} \int_{0}^{\infty} c^{2} \chi \zeta_{R} d x d t \leq b_{0} \alpha A\left(D, b_{0}, \alpha\right) \sqrt{T} .
\end{aligned}
$$

Next we remark that the term

$$
\int_{0}^{T} \int_{R}^{2 R}\left\{\frac{c^{2}}{2}\right\}_{x} \zeta_{R}^{\prime} d x d t=-\int_{0}^{T} \int_{R}^{2 R}\left\{\frac{c^{2}}{2}\right\} \zeta_{R}^{\prime \prime} d x d t
$$

tends to zero as $R$ tends to infinity. Therefore letting $R$ tend to infinity in (19) and applying the Lebesgue monotone convergence theorem, we deduce the result of Lemma 3.2.

Lemma $3.3 c-\psi$ is nonincreasing in time.

Proof For $h \geq 0$, let $y_{h}$ be defined by $y_{h}(x, t)=c(x, t+h)-\psi(x, t+h)$. Since $c \leq \psi$ we know that

$$
y_{h}(x, 0) \leq 0
$$

We have

$$
y_{h t}=D y_{h x x}-c(x, t+h) \chi(x, t+h) .
$$

Note that $\chi$ is nondecreasing in $t$. It follows that

$$
\begin{aligned}
\left(y_{h}-y_{0}\right)_{t}=D\left(y_{h}-y_{0}\right)_{x x}-c(x, t+h) \chi(x, t+h)+c(x, t) \chi(x, t) \\
\leq D\left(y_{h}-y_{0}\right)_{x x}-[c(x, t+h)-c(x, t)] \chi(x, t) \\
=D\left(y_{h}-y_{0}\right)_{x x}-[\psi(x, t+h)-\psi(x, t)] \chi(x, t) \\
\quad-[c(x, t+h)-\psi(x, t+h)-c(x, t)+\psi(x, t)] \chi(x, t) .
\end{aligned}
$$

Because $\psi$ is nondecreasing in $t$, we obtain that

$$
\left(y_{h}-y_{0}\right)_{t} \leq D\left(y_{h}-y_{0}\right)_{x x}-\left(y_{h}-y_{0}\right) \chi(x, t) .
$$

Next, we multiply by $\left(y_{h}-y_{0}\right)^{+}$, integrate by parts, and use (20).

Remark 3.4 Lemma 3.3 implies that $c$ is nonincreasing in $t$ when $x \leq \alpha \sqrt{t}$. 
We start with a discussion of the first precipitation band. To be precise, we define

$$
\mathcal{B}(t)=\{x \geq 0: w(y, t)>0 \text { for all } 0 \leq y<x\} .
$$

First, we show the existence of such set, under appropriate conditions.

Lemma 3.5 Define $S(t)=\max \{x: x \in \mathcal{B}(t)\}$ if $\mathcal{B}(t) \neq \varnothing$ and $S(t)=0$ otherwise. If $A\left(D, b_{0}, \alpha\right)>C_{s}$, then $S(t)>0$ for all $t>0$. Moreover, $S(\cdot)$ is nondecreasing.

Proof Clearly,

$$
c_{t} \geq D c_{x x}+\frac{b_{0} \alpha}{2 \sqrt{t}} \delta(x-\alpha \sqrt{t})-c \geq D c_{x x}+\frac{b_{0} \alpha}{2 \sqrt{t}} \delta(x-\alpha \sqrt{t})-A\left(D, b_{0}, \alpha\right) .
$$

Consequently,

$$
\left[c+A\left(D, b_{0}, \alpha\right) t\right]_{t} \geq D\left[c+A\left(D, b_{0}, \alpha\right) t\right]_{x x}+\frac{b_{0} \alpha}{2 \sqrt{t}} \delta(x-\alpha \sqrt{t})
$$

and $c \geq \psi-A\left(D, b_{0}, \alpha\right) t$. So, if $A\left(D, b_{0}, \alpha\right)>C_{s}$ and if $t$ is sufficiently small, then $c(x, t)>C_{s}$ in the region $0 \leq x<\alpha \sqrt{t}$. The lemma follows immediately.

Lemma 3.6 Suppose that $t$ is such that $S(t+\epsilon)>S(t)$ for all $\epsilon>0$. Then $c(S(t), t)=C_{s}$.

Proof This follows from the continuity of $c$.

Lemma $3.7 S$ is continuous on $[0, \infty)$.

Proof Suppose, to the contrary, that $x_{1}:=S\left(t^{*-}\right)<S\left(t^{*+}\right):=x_{2}$ for some $t^{*} \geq 0$. Then for every $x \in\left(x_{1}, x_{2}\right)$ it holds that:

(1) $c(x, t) \leq C_{s}$ for $t \leq t^{*}$, and

(2) there is a sequence $t_{1}>t_{2}>\cdots \rightarrow t^{*}$ such that $c\left(x, t_{i}\right)>C_{s}$.

We deduce that $c\left(x, t^{*}\right)=C_{s}$ for $x_{1}<x<x_{2}$ and, by Lemma 3.3, that $\alpha \sqrt{t^{*}} \leq x_{1}$. Choose $x_{3}=\frac{x_{1}+x_{2}}{2}$. Clearly, $c\left(x_{3}, t^{*}\right)=C_{s}$ and $c$ assumes its maximum over the region $\left[x_{1}, \infty\right) \times$ $\left[0, t^{*}\right]$ in the point $\left(x_{3}, t^{*}\right)$. By the maximum principle we deduce that $c \equiv C_{s}$ in $\left[x_{3}, \infty\right) \times$ $\left[0, t^{*}\right]$, which is in contradiction with the initial condition $c(x, 0)=0$ (note that the curve $\{x=\alpha \sqrt{t}\}$ does not enter this region).

In what follows, we shall prove that, under the additional technical condition that $2 C_{s}>$ $A\left(D, b_{0}, \alpha\right)$, there is a time $\hat{t}$ such that $S(t)=s(\hat{t})$ for all $t>\hat{t}$. That is: after some time the first "precipitation region" (that is, a region where $w>0$ ) does not grow further. Assuming an additional property of the weak solution, it is not difficult to show that this implies that a new precipitation region must come into existence if $D, \alpha, b_{0}$ and $C_{s}$ are appropriate. The proof which we are going to present can be used for the subsequent precipitation regions as well, so that we are able to prove the existence of an infinite sequence of such regions (provided that our assumption holds true).

Lemma 3.8 For any $\gamma>0$, there exists a time $t>0$ such that $c(S(t), t)<\gamma$. 
Proof Suppose that on the contrary there exists $\gamma>0$ such that $c(S(t), t) \geq \gamma$ for all $t>0$. Let $\delta>0$ and $\tilde{t}>0$ be such that $S(t)>\delta$ when $t>\tilde{t}$, see Lemma 3.5. By the CauchySchwarz inequality it holds that, when $0 \leq S(t)-\delta \leq x \leq S(t)$,

$$
[c(S(t), t)-c(x, t)]^{2} \leq(S(t)-x) \int_{x}^{S(t)} c_{\xi}(\xi, t)^{2} d \xi \leq(S(t)-x) \int_{0}^{\infty} c_{\xi}(\xi, t)^{2} d \xi .
$$

For $T>\tilde{t}$ we obtain

$$
\frac{1}{T} \int_{\tilde{t}}^{T}[c(S(t), t)-c(x, t)]^{2} d t \leq \frac{S(t)-x}{T} \int_{0}^{T} \int_{0}^{\infty} c_{\xi}(\xi, t)^{2} d \xi d t,
$$

which we integrate with respect to $x$ over $\{S(t)-\delta<x<S(t)\}$ to obtain

$$
\frac{1}{T} \int_{\tilde{t}}^{T} \int_{S(t)-\delta}^{S(t)}[c(S(t), t)-c(x, t)]^{2} d x d t \leq \frac{\delta^{2}}{2 T} \int_{0}^{T} \int_{0}^{\infty} c_{\xi}(\xi, t)^{2} d \xi d t .
$$

By Lemma 3.2, the right-hand side of this expression tends to 0 as $T \rightarrow \infty$. Therefore,

$$
\left.\frac{1}{T} \int_{\tilde{t}}^{T} \int_{S(t)-\delta}^{S(t)}\left[c(S(t), t)^{2}-2 c(S(t), t) c(x, t), t\right)+c(x, t)^{2}\right] d x d t \rightarrow 0 \quad \text { as } T \rightarrow \infty .
$$

However, we also have that

$$
\liminf _{T \rightarrow \infty} \frac{1}{T} \int_{\tilde{t}}^{T} \int_{S(t)-\delta}^{S(t)}[c(S(t), t)]^{2} d x d t \geq \delta \gamma^{2} .
$$

Moreover we deduce from Lemma 3.2 that

$$
\frac{1}{T} \int_{\tilde{t}}^{T} \int_{S(t)-\delta}^{S(t)} \chi(x, t)[c(x, t)]^{2} d x d t \rightarrow 0 \quad \text { as } T \rightarrow \infty .
$$

Note that Lemma 3.6 implies that, for any $x<S(t)$, it holds that $w(x, t)>0$ and therefore that $\chi(x, t)=1$. It follows that

$$
\frac{1}{T} \int_{\tilde{t}}^{T} \int_{S(t)-\delta}^{S(t)}[c(x, t)]^{2} d x d t \rightarrow 0 \quad \text { as } T \rightarrow \infty .
$$

Finally, by the Cauchy-Schwarz inequality, we get

$$
\frac{1}{T} \int_{\tilde{t}}^{T} \int_{S(t)-\delta}^{S(t)} \chi(x, t) c(x, t) d x d t \leq \frac{1}{T} \sqrt{\delta(T-\tilde{t})} \sqrt{\int_{\tilde{t}}^{T} \int_{S(t)-\delta}^{S(t)} \chi(x, t)[c(x, t)]^{2} d x d t}
$$

which by Lemma 3.2 implies that

$$
\frac{1}{T} \int_{\tilde{t}}^{T} \int_{S(t)-\delta}^{S(t)} c(x, t) d x d t \leq C T^{\frac{-1}{4}} \rightarrow 0 \quad \text { as } T \rightarrow \infty .
$$

Thus

$$
\frac{1}{T} \int_{\tilde{t}}^{T} \int_{S(t)-\delta}^{S(t)} c(S(t), t) c(x, t) d x d t \leq A\left(D, b_{0}, \alpha\right) \frac{1}{T} \int_{\tilde{t}}^{T} \int_{S(t)-\delta}^{S(t)} c(x, t) d x d t
$$


which tends to zero as $T \rightarrow \infty$, so that we have reached a contradiction. This completes the proof.

Lemma 3.9 If $S(\hat{t}) \leq \alpha \sqrt{\hat{t}}$ for some $\hat{t}>0$, then $S(t)=S(\hat{t})$ for all $t>\hat{t}$.

Proof When $\hat{x}:=S(\hat{t}) \leq \alpha \sqrt{\hat{t}}$, then Lemma 3.3 implies that $c_{t}(\hat{x}, t) \leq 0$ for all $t>\hat{t}$. Since $c(\hat{x}, t) \leq C_{s}$ for all $t \leq \hat{t}$ we deduce that $c(\hat{x}, t) \leq C_{s}$ for all $t>0$, so that $S(\cdot)$ cannot grow further.

Theorem 3.10 Provided that $2 C_{s}>A\left(D, b_{0}, \alpha\right)$, there is a $T^{*}>0$ such that $S(t)=S\left(T^{*}\right)$ for every $t>T^{*}$.

Proof Let $\beta>0$ be defined by the relation $\Psi(\beta)=C_{s}$. It is clear that $\psi(x, t) \geq C_{s}$ whenever $x \leq \beta \sqrt{t}$. Moreover, from Theorem 2.7 we deduce that $S(t) \leq \beta \sqrt{t}$, so that

$$
\psi(x, t) \geq C_{s} \quad \text { whenever } x \leq S(t) .
$$

Choose $0<\gamma<2 C_{s}-A\left(D, b_{0}, \alpha\right)$ and apply Lemma 3.8 to obtain a time $T^{*}$ such that $c\left(S\left(T^{*}\right), T^{*}\right)<\gamma<C_{s}$. We claim that $T^{*}$ satisfies the requirements of the theorem. To see this, suppose, for contradiction, that there is a $t^{*}>T^{*}$ such that $S\left(t^{*}\right)>S\left(T^{*}\right)$. Then, since by Lemma $3.7 S$ is continuous, it must continuously grow from $S\left(T^{*}\right)$ to $S\left(t^{*}\right)$, and before it grows it may remain constant for some time, namely, there exists a time $\bar{t} \in\left(T^{*}, t^{*}\right)$ such that

$$
S(\bar{t})=S\left(T^{*}\right) \quad \text { for every } T^{*} \leq t \leq \bar{t} .
$$

At the time $\bar{t}$ when it starts to grow, then by Lemma 3.6 the concentration value on $S$ has to be equal to $C_{s}$, namely

$$
c\left(S\left(T^{*}\right), \bar{t}\right)=C_{s} .
$$

By (21), $c\left(S\left(T^{*}\right), T^{*}\right)-\psi\left(S\left(T^{*}\right), T^{*}\right)<\gamma-C_{s}$. But now, Lemma 3.3 implies that

$$
c\left(S\left(T^{*}\right), \bar{t}\right)-\psi\left(S\left(T^{*}\right), \bar{t}\right)=C_{s}-\psi\left(S\left(T^{*}\right), \bar{t}\right) \leq c\left(S\left(T^{*}\right), T^{*}\right)-\psi\left(S\left(T^{*}\right), T^{*}\right)<\gamma-C_{s} .
$$

The choice of $\gamma$ implies that $\psi\left(S\left(T^{*}\right), \bar{t}\right)>2 C_{s}-\gamma>A\left(D, b_{0}, \alpha\right)$, which is a contradiction.

Theorem 3.10 states that $S$ comes to a halt, eventually. But it does not exclude the possibility that $S(t)=S\left(T^{*}\right)$ for every $t>T^{*}$ and that, for $x>S\left(T^{*}\right)$ and $x-S\left(T^{*}\right)$ sufficiently small, there is a time $t>T^{*}$ such that $c(x, t)>C_{s}$. That is: if $t$ is sufficiently large, the line $\left\{\left(S\left(T^{*}\right), t\right)\right\} \subset \mathbb{R}^{2}$ separates two regions where precipitations have occurred, whereas the line itself is "precipitation-free". In order to deal with that situation, we define

$$
\begin{aligned}
& \overline{\mathcal{B}}(t)=\{x \geq 0: w(y, t)>0 \text { for almost all } 0 \leq y<x\}, \quad \text { and } \\
& \bar{S}(t)=\max \{x: x \in \overline{\mathcal{B}}(t)\} \quad \text { if } \overline{\mathcal{B}}(t) \neq \emptyset ; \quad \bar{S}(t)=0 \quad \text { otherwise. }
\end{aligned}
$$

Lemma 3.11 If $\bar{S}(\hat{t})<\alpha \sqrt{\hat{t}}$ for some $\hat{t}>0$, then $\bar{S}(t)=\bar{S}(\hat{t})$ for all $t>\hat{t}$.

Proof For $x \geq \bar{S}(\hat{t})$ sufficiently close to $\bar{S}(\hat{t})$ it holds that $x<\alpha \sqrt{t}$ for all $t \geq \hat{t}$. Remark 3.4 completes the proof. 
Theorem 3.12 Provided that $2 C_{s}>A\left(D, b_{0}, \alpha\right)$, there is a $T^{\#}>0$ such that $\bar{S}(t)=\bar{S}\left(T^{\#}\right)$ for every $t>T^{\#}$.

Proof Suppose, to the contrary, that for all $t$ there is a $\tilde{t}>t$ such that $\bar{S}(\tilde{t})>\bar{S}(t)$. Then Remark 3.4 implies that $\bar{S}(t) \geq \alpha \sqrt{t}$ for all $t>0$. Next we choose $0<\gamma<2 C_{s}-A\left(D, b_{0}, \alpha\right)$ and apply the arguments of Lemma 3.8 to deduce that there exists a time $T^{\#}$ such that $c\left(\bar{S}\left(T^{\#}\right), T^{\#}\right)<\gamma$. Note that $\gamma<C_{s}$. Using that $\alpha \sqrt{t} \leq \bar{S}\left(T^{\#}\right)$ for all $t \leq T^{\#}$, we easily deduce from the maximum principle that $c\left(x, T^{\#}\right)<C_{s}$ for all $x \geq \bar{S}\left(T^{\#}\right)$. The same argument as the one used in the proof of Theorem 3.10 yields that there exists an $\epsilon>0$ such that, for all $t>T^{\#}, c\left(\bar{S}\left(T^{\#}\right), t\right)<C_{s}-\epsilon$. In particular, if $\alpha \sqrt{t^{\#}}=\bar{S}\left(T^{\#}\right)$, we find that $t^{\#} \geq T^{\#}$ and we obtain, again by the maximum principle, that there exists $\delta>0$ such that $c<C_{s}-\delta$ in the set $\left\{(x, t): x \geq \bar{S}\left(T^{\#}\right), T^{\#} \leq t \leq t^{\#}\right\}$. We claim that there exists $t^{*}>t^{\#}$ such that $c<C_{s}$ in the set $\left\{(x, t): x \geq \bar{S}\left(T^{\#}\right), t^{\#} \leq t<t^{*}\right\}$. Otherwise, there would exist sequences $\left\{x_{i} \geq \bar{S}\left(T^{\#}\right)\right\}$ and $\left\{t_{i} \in\left[t^{\#}, t^{\#}+1\right]\right\}$ such that $t_{i} \downarrow t^{\#}$ and $c\left(x_{i}, t_{i}\right) \geq C_{s}$. Since $C_{s} \leq c\left(x_{i}, t_{i}\right) \leq \Psi\left(\frac{x_{i}}{\sqrt{t_{i}}}\right)$, it would follow that $\bar{S}\left(T^{\#}\right) \leq x_{i} \leq \beta \sqrt{t_{i}} \leq \beta \sqrt{t^{\#}+1}$, with $\beta$ as in the proof of Theorem 3.10. Then there would exist $\bar{x}$ and a subsequence of $\left\{x_{i}\right\}$ which we denote again by $\left\{x_{i}\right\}$ such that $x_{i} \rightarrow \bar{x}$. By continuity, this would lead to a contradiction. Using Remark 3.4 we deduce that, for all $\bar{S}\left(T^{\#}\right)<x<\alpha \sqrt{t^{*}}$, it holds that $w(x, t)=0$ for all $t>T^{\#}$.

We shall now establish conditions for the existence of infinitely many distinct precipitation bands. We do that under the additional:

Assumption $\mathcal{X}(x, t)=0$ whenever $\int_{0}^{t}\left(c(x, \tau)-C_{s}\right)^{+} d \tau=0$.

Theorem 3.13 If $2 C_{s}>A\left(D, b_{0}, \alpha\right)>C_{s}$ and if $\frac{\alpha}{\sqrt{D}}$ is sufficiently large, then there are infinitely many distinct precipitation regions.

Proof Let $T^{\#}$ be as in Theorem 3.12. By Lemma 3.7, we may suppose that $T^{\#}$ is minimal. Set $\bar{x}:=\bar{S}\left(T^{\#}\right)$. By Remark 3.4 and the minimality of $T^{\#}$ we have that $\bar{x} \geq \alpha \sqrt{T^{\#}}$; consequently, there is a $t^{*} \geq T^{\#}$ such that $\alpha \sqrt{t^{*}}=\bar{x}$. We shall first prove that a second precipitation region must exist. To that purpose we suppose that, on the contrary, $c(x, t) \leq C_{s}$ for all $x>\bar{x}, t>0$. Then $c$ satisfies

$$
\left(\mathrm{P}^{*}\right) \begin{cases}c_{t}=D c_{x x}+\frac{b_{0} \alpha}{2 \sqrt{t}} \delta(x-\alpha \sqrt{t}), & x>\bar{x}, t>t^{*}, \\ c(\bar{x}, t) \geq 0, & t>t^{*} \\ c\left(x, t^{*}\right) \geq 0, & x>\bar{x}\end{cases}
$$

Upon the transformation of variables

$$
\xi=\frac{x}{\sqrt{t}} ; \quad \tau=\log t ; \quad \tilde{c}(\xi, \tau)=c(x, t)
$$

and omitting the tilde again, we deduce that $c$ is a solution of

$$
\left(\mathrm{P}^{\#}\right) \quad \begin{cases}c_{\tau}=D c_{\xi \xi}+\frac{\xi}{2} c_{\xi}+\frac{b_{0} \alpha}{2} \delta(\xi-\alpha), & \xi>\bar{x} e^{-\tau / 2}, \tau>\log t^{*}, \\ c\left(\bar{x} e^{-\tau / 2}, \tau\right) \geq 0, & \tau>\log t^{*} \\ c\left(\xi, \log t^{*}\right) \geq 0, & \xi>\frac{\bar{x}}{\sqrt{t^{*}}}(=\alpha) .\end{cases}
$$


The maximum principle implies that $c(\xi, \tau) \geq 0$ for all $(\xi, \tau)$ with $\xi \geq \bar{x} e^{-\tau / 2}, \tau \geq \log t^{*}$. For $\hat{\tau}>0$ given, let $\tau^{*}=\log t^{*}+\hat{\tau}$ and $\xi^{*}=\bar{x} e^{-\tau^{*} / 2}$; we have that

$$
c(\xi, \tau) \geq 0 \text { for all }(\xi, \tau) \text { with } \xi \geq \xi^{*}, \tau \geq \tau^{*}
$$

Note that $\xi^{*}<\alpha$. We obtain that $c \geq z$ in the region $\xi \geq \xi^{*}, \tau \geq \tau^{*}$, where $z$ satisfies

$$
(\overline{\mathrm{P}}) \begin{cases}z_{\tau}=D z_{\xi \xi}+\frac{\xi}{2} z_{\xi}+\frac{b_{0} \alpha}{2} \delta(\xi-\alpha), & \xi>\xi^{*}, \tau>\tau^{*}, \\ z\left(\xi^{*}, \tau\right)=0, & \tau>\tau^{*}, \\ z\left(\xi, \tau^{*}\right)=0, & \xi>\xi^{*} .\end{cases}
$$

The maximum principle implies that $z \geq 0$ and it is easy to see that $z_{h}(\xi, \tau):=z(\xi, \tau+h) \geq$ $z(\xi, \tau)$ for $h>0$. Thus $z$ is nondecreasing in $\tau$ and since it is bounded from above by $\Psi$, we deduce that, as $\tau \rightarrow \infty, z$ tends to the steady state $\bar{z}$ defined by

$$
D \bar{z}_{\xi \xi}+\frac{\xi}{2} \bar{z}_{\xi}+\frac{b_{0} \alpha}{2} \delta(\xi-\alpha)=0 ; \quad \bar{z}\left(\xi^{*}\right)=\bar{z}(\infty)=0
$$

It is easy to obtain $z$ explicitly. It holds in particular that

$$
\begin{aligned}
\|\bar{z}\|_{\infty} & =\bar{z}(\alpha)=B\left(D, b_{0}, \alpha, \xi^{*}\right):=A\left(D, b_{0}, \alpha\right) \frac{\int_{\xi^{*}}^{\alpha} e^{-\eta^{2} / 4 D} d \eta}{\int_{\xi^{*}}^{\infty} e^{-\eta^{2} / 4 D} d \eta} \\
& =A\left(D, b_{0}, \alpha\right) \frac{\int_{\xi^{*} / \sqrt{D}}^{\alpha / \sqrt{D}} e^{-s^{2} / 4} d s}{\int_{\xi^{*} / \sqrt{D}}^{\infty} e^{-s^{2} / 4} d s} .
\end{aligned}
$$

Note that, given $D$, we can choose $\hat{\tau}$ so large that $\frac{\xi^{*}}{\sqrt{D}}$ is arbitrarily close to 0 . Clearly, $B\left(D, b_{0}, \alpha, \xi^{*}\right)$ is arbitrarily close to $A\left(D, b_{0}, \alpha\right)$ once $\frac{\alpha}{\sqrt{D}}$ and $\hat{\tau}$ are sufficiently large (recall that $A\left(D, b_{0}, \alpha\right)$ remains bounded as $\alpha \rightarrow \infty$, see Lemma 3.1). It follows from Theorem 3.12 that, under the conditions of Theorem 3.13, a second precipitation region must exist. That is, the assumption that no second precipitation region exists leads to a contradiction.

Similarly to the first precipitation region, the second one (and all subsequent ones) will also be bounded in the $x$-direction, given that $2 C_{s}>A\left(D, b_{0}, \alpha\right)$; this is also valid for the "generalized" precipitation regions where $w>0$ almost everywhere. To prove the occurrence of subsequent precipitations we use the same arguments as above; we remark that the essential ingredient of the proof has been the existence of $\xi^{*}$ such that $B\left(D, b_{0}, \alpha, \xi^{*}\right)$ is sufficiently close to $A\left(D, b_{0}, \alpha\right)$. It is clear from the above construction and (22) that this can be again realized provided that $\frac{\alpha}{\sqrt{D}}$ is sufficiently large, independently of the value of $\bar{x}$. Thus, our conditions guarantee the existence of infinitely many, distinct, precipitation regions.

Remark 3.14 It is easy to see, by the maximum principle, that if a new precipitation region germinates at $(x, t)$, we must have that $x=\alpha \sqrt{t}$. By Lemma 3.3 (or Remark 3.4) we deduce that, in the $(x, t)$ plane, such a precipitation region can only extend to the right of the point of initiation. 


\section{Discussion}

In this paper we have performed the mathematical study of a one-dimensional Keller and Rubinow model for Liesegang bands; under suitable assumptions, we prove the existence of Liesegang bands and justify the time law.

Discussing about quantitative properties of patterns in problems involving sharp fronts is technically easier than dealing with diffusive fronts with a certain width. This leads us to first take the singular limit of part of the Keller and Rubinow system in order to obtain a sharp interface system. This subsystem describes an irreversible chemical reaction for two species of concentrations $a_{k}$ and $b_{k}$. Chemical arguments imply that the reaction rate $k$ is very large and it has been shown previously that, as $k \rightarrow \infty, a_{k}$ and $b_{k}$ converge to limit profiles $\bar{a}$ and $\bar{b}$, in such a way that $\bar{a} \bar{b}=0$ and that $k a_{k} b_{k}$ tends to a $\delta$-distribution moving with the velocity $\sim \frac{1}{\sqrt{t}}$. In turn this moving $\delta$-interface acts as a source term in a reaction-diffusion equation for a third species of concentration $c$, which makes it quite natural to derive the time law. Eventually, we obtain a limiting Keller and Rubinow model for which we prove that the time law is satisfied, by means of arguments essentially based upon the maximum principle.

This singular limit procedure is a kind of a mathematical idealization in which the reaction between $a_{k}$ and $b_{k}$ is modeled to occur in an infinitely narrow interface, whereas in the real physical context this reaction occurs throughout space. Actually, outside a certain narrow region, namely the interface, which has a width of the order of, say, the size of a few molecules, the reaction has almost finished or is still in a very initial state. This idealization brings a technical difficulty from the mathematical viewpoint, namely a "discontinuity" in the system at the moment that the super-saturation density is achieved and that the precipitation occurs. In particular we cannot prove the uniqueness of the solution, and one cannot prove either the spacing law except if one makes extra assumptions on the solution profile at the precipitation times. We refer to Theorem 3.6 by [8], where they show the spacing law under the assumption that the solution profiles at each precipitation time are approximately the same. This property is indeed satisfied in our numerical simulations and the spacing law holds, as it is shown in the numerical simulations of Fig. 4.

On the other hand, things are slightly different for the width law. In fact, we know from numerical evidence that the width law is neither valid in the Keller and Rubinow model nor in the sharp interface limit. In particular the width seems to strongly depend upon the physical properties of the colloids. Moreover it clearly appears in numerical simulations that this model does not permit to represent Liesegang bands nor rings in higher space dimension. Therefore, we should probably analyse the way in which the precipitation of colloids occurs in more details in order to be able to derive a new model. Mimura, Ohnishi, and Ueyama [7] attempt to construct such a model; to that purpose they thoroughly study the precipitation procedure by means of the physical theory for the creation of colloids. Kai and Muller [4], and Kai [3] state that the width law is often not satisfied: occasionally the width varies randomly, and it even satisfies a very different law in a special case. The width law probably depends on which materials or matrix substrates are used in the chemical experiments. Therefore, we should construct a model which varies according to the experiment. Future work will involve searching for and studying such a new model which should not only be valid in higher space dimension, but also be suitable in special cases of interest. However it is already interesting to understand the Keller and Rubinow model which both contains the time and spacing laws.

Acknowledgements The authors are grateful to Prof. M. Oliver (Jacobs University, Bremen, Germany) for valuable discussions. The remarks of the referees have been very useful. 
Open Access This article is distributed under the terms of the Creative Commons Attribution Noncommercial License which permits any noncommercial use, distribution, and reproduction in any medium, provided the original author(s) and source are credited.

\section{References}

1. Hilhorst, D., van der Hout, R., Peletier, L.A.: The fast reaction limit for a reaction-diffusion system. J. Math. Anal. Appl. 199, 349-373 (1996)

2. Hilhorst, D., van der Hout, R., Peletier, L.A.: Diffusion in the presence of fast reaction: the case of a general monotone reaction term. J. Math. Sci. Univ. Tokyo 4, 469-517 (1997)

3. Kai, S.: Private communications (2006)

4. Kai, S., Muller, S.C.: Spatial and temporal macroscopic structures in chemical reaction system: precipitation patterns and interfacial motion. Sci. Form 1, 8-38 (1985)

5. Keller, J.B., Rubinow, S.I.: Recurrent precipitation and Liesegang rings. J. Chem. Phys. 74, 5000-5007 (1981)

6. Liesegang, R.E.: Chemische Fernwirkung. Photo. Archiv 800, 305-309 (1896)

7. Mimura, M., Ohnishi, I., Ueyama, D.: A mathematical aspect of Liesegang phenomena in two space dimensions. Res. Rep. Res. Inst. Math. Sci. 1499, 185-201 (2006)

8. Ohnishi, I., Mimura, M.: A mathematical aspect of Liesegang phenomena. In: Proceedings of Equadiff-11, pp. 343-352 (2005)

9. Smith, D.: On Ostwald's supersaturation theory of rhythmic precipitation (Liesegang's rings). J. Chem. Phys. 81, 3102-3115 (1984) 